\title{
Familial co-aggregation of schizophrenia and eating disorders in Sweden and Denmark
}

\author{
Ruyue Zhang $\mathbb{D}^{1} \cdot$ Janne Tidselbak Larsen $\mathbb{1}^{2,3,4} \cdot$ Ralf Kuja-Halkola $\mathbb{D}^{1} \cdot$ Laura Thornton ${ }^{5}$. Shuyang Yao $\mathbb{B}^{1} \cdot$ \\ Henrik Larsson ${ }^{1,6} \cdot$ Paul Lichtenstein $\mathbb{1}^{1} \cdot$ Liselotte Vogdrup Petersen ${ }^{2,3} \cdot$ Cynthia M. Bulik $\mathbb{D}^{1,5,7} \cdot$ Sarah E. Bergen $\left(\mathbb{D}^{1}\right.$
}

Received: 22 September 2019 / Revised: 10 April 2020 / Accepted: 20 April 2020

\begin{abstract}
Eating disorders and schizophrenia are both moderately to highly heritable and share significant genetic risk despite distinct diagnostic criteria. Large-scale family studies on the co-aggregation of these disorders are lacking. Thus, we aimed to estimate the co-occurrence and familial co-aggregation of these disorders within the entire Swedish and Danish population. The proband cohort consisted of individuals born in Sweden (1977-2003) and Denmark (1984-2006) and still residing in their respective country at age six $\left(N_{\text {Sweden }}=2,535,191, N_{\text {Denmark }}=1,382,367\right)$. Probands were linked to their biological parents, siblings, grandparents, uncles/aunts, and cousins. Diagnoses for anorexia nervosa (AN) and other eating disorders (OED: bulimia nervosa, binge-eating disorder, and eating disorder not otherwise specified) for probands and schizophrenia diagnoses for both probands and relatives were obtained. The likelihood of having schizophrenia in those with AN or OED and their relatives was compared with individuals without eating disorder diagnoses and their relatives. Probands with AN or OED were more likely to have schizophrenia than probands without these disorders. All relatives of probands with AN or OED (except parents and uncles/aunts of probands with AN) were at increased risk of schizophrenia. In general, the magnitude of odds ratios attenuated with decreasing genetic relatedness. These results suggest familial liability contributes to the association between eating disorders and schizophrenia. Clinicians should be mindful of this comorbid and co-aggregation pattern as it may influence case conceptualization and treatment decisions.
\end{abstract}

Supplementary information The online version of this article (https:// doi.org/10.1038/s41380-020-0749-x) contains supplementary material, which is available to authorized users.

Sarah E. Bergen

sbergen@gmail.com

1 Department of Medical Epidemiology and Biostatistics, Karolinska Institutet, Stockholm, Sweden

2 National Centre for Register-based Research, Aarhus University, Aarhus, Denmark

3 iPSYCH, The Lundbeck Foundation Initiative for Integrative Psychiatric Research, Aarhus, Denmark

4 Centre for Integrated Register-based Research at Aarhus University (CIRRAU), Aarhus, Denmark

5 Department of Psychiatry, University of North Carolina at Chapel Hill, Chapel Hill, NC, USA

6 School of Medical Sciences, Örebro University, Örebro, Sweden

7 Department of Nutrition, University of North Carolina at Chapel Hill, Chapel Hill, NC, USA

\section{Introduction}

Schizophrenia is a severe psychiatric disorder characterized by disordered thoughts, perceptions, and behaviors with onset typically in late adolescence and early adulthood, affecting about 21 million people worldwide [1, 2]. Eating disorders, including anorexia nervosa (AN), bulimia nervosa, and binge-eating disorder, are also serious disorders usually arising in adolescence and early adulthood. Both schizophrenia and eating disorders are associated with considerable somatic and psychiatric morbidity, as well as elevated mortality rates [3-5].

Schizophrenia and eating disorders are highly heritable conditions with twin-based heritability estimates of $64-81 \%$ for schizophrenia and 50-83\% for eating disorders [6-9]. Despite the distinct diagnostic criteria of these disorders, significant positive single-nucleotide polymorphism-based genetic correlations between schizophrenia and AN have been reported in previous studies $\left(r_{\mathrm{g}}=0.19-0.29\right)$ [10,11]. Although a small literature explores comorbidity between eating disorders and schizophrenia [12-15], almost no 
research has been conducted examining the co-occurrence of schizophrenia and eating disorder diagnoses within individuals and co-aggregation of these disorders in families using population-based data.

However, relationships between symptoms of eating disorders and schizophrenia have been explored in a few epidemiological investigations. Eating disturbances (e.g., pica, faddiness, refusal) in childhood were found to be associated with schizophrenia in adulthood in a small case-control study [16]. Psychotic experiences during early adolescence were associated with increased risk for disordered eating behaviors such as binge eating, purging, and fasting in late adolescence in a UK birth cohort [17]. Moreover, an association between having a first-degree relative with schizophrenia and the lifetime risk for bulimia nervosa was reported in the National Survey of American Life [18]. However, since these results were primarily based on small clinical samples or self-reported data, it remains unclear if the co-occurrence of clinically diagnosed eating disorders and schizophrenia, as well as the familial co-aggregation of these disorders is present at the population level.

The genetic association between schizophrenia and eating disorders other than AN is still unknown, and whether these disorders have common familial risk is unclear. Comparing co-aggregation of disorders across different types of relatives can provide information on the extent to which the degree of relatedness influences the risk of schizophrenia. Accordingly, additional investigation into the nature and impact of the shared genetic etiology between schizophrenia and eating disorders is merited.

The primary aim of this study was to estimate the co-occurrence and familial co-aggregation between schizophrenia and eating disorders in the Swedish and Danish populations.

\section{Materials and methods}

The study has been approved by the Regional Ethics Review Board in Stockholm, Sweden (Dnr 2013/862-31/5) and the Health Data Protection Agency in Denmark (FSEID00000098). The study used population-based registers, and no individuals in the study populations were identifiable at any time.

\section{Data sources}

Sweden: unique personal identification numbers were used to link several Swedish registers. We obtained sex and birth year information from the Swedish Total Population Register [19]. Information regarding stillbirths and congenital malformations was acquired from the Swedish Medical Birth Register [20]. Dates and causes of death, when applicable, were obtained from the Swedish Cause of Death Register (CDR). We received immigration and emigration data via the Swedish Migration Register. Using the Swedish Multi-Generation Register [19], we linked individuals (probands) to their biological parents and other relatives. Records of psychiatric inpatient (since 1973) and outpatient (since 2001) contacts from across Sweden were obtained from the Swedish National Patient Register (NPR, based on the International Classification of Diseases (ICD)) [21]. We also derived eating disorder diagnoses from two other Swedish National Quality Registers [Riksät and Stepwise, based on the Diagnostic and Statistical Manual of Mental Disorders, 4th Edition, Text Revision, DSM-IV-TR [22]] [23]. Death due to eating disorders or schizophrenia obtained from the Swedish CDR also constituted diagnoses.

Denmark: we obtained sex, birth year, information on parental IDs, and continuously updated information on places of residence and vital status from the Danish Civil Registration System (CRS) [24]. Relatives were identified through CRS. Unique personal identification numbers were used to link all Danish national registers. Congenital malformation information was acquired from the Danish Medical Birth Register [25]. Adoptive information came from Statistics Denmark [26]. Records of all admissions to Danish psychiatric inpatient facilities (since 1970) were taken from the Danish Psychiatric Central Research Register (PCRR, based on ICD) [27]. We also obtained diagnostic records on all inpatient (since 1977) and outpatient (since 1995) admissions to public hospitals from the Danish NPR [28]. Death due to eating disorders or schizophrenia as recorded in the Danish CDR also constituted diagnoses.

\section{Study population}

The Swedish proband cohort included individuals born between January 1, 1977 and December 31, 2003 (3,469,851 individuals) with the following exclusions: stillbirths and congenital malformations $(n=105,642)$, death before 6th birthday $(n=12,988)$, emigration before 6th birthday ( $n=$ $82,229)$, birth outside of Sweden/immigration $(n=708,335)$, no parental information $(n=20,733)$, and adopted children $(n=4733)$, yielding a final sample of $2,535,191$ individuals. We followed the study population until December 31, 2013, when the youngest individuals were 10 years old and the oldest individuals were 36 .

The Danish proband cohort included individuals born between January 1, 1984 and December 31, 2006 $(1,945,191$ individuals) with the following exclusions: congenital malformations $(n=47,924)$, death prior to 6 th birthday $(n=10,196)$, emigration prior to 6th birthday $(n=$ $25,414)$, disappearance prior to 6th birthday $(n=694)$, birth outside of Denmark/immigration $(n=462,057)$, no parental information $(n=11,203)$, and adopted children $(n=5336)$, 
yielding a final sample of 1,382,367 individuals. We followed the study population until December 31, 2016, when the youngest individuals were 10 years old and the oldest individuals were 32 .

Finally, using the Swedish Multi-Generation Register and Danish CRS, we identified six cohorts of biological relatives representing decreasing levels of shared genetic and environmental relatedness: parents, full siblings (excluding monozygotic twins in Sweden and excluding all twins in Denmark as zygosity was not available in the registers to which we had access), grandparents, half siblings, uncles/aunts (full siblings of probands' biological parents), full cousins (biological children of defined uncles/aunts). For all of the relatives related to the probands, individuals born before January 1, 2008 in Sweden and born before January 1, 2011 in Denmark were included. All aforementioned relatives (except grandparents) without parental information were excluded. Immigrants as well as individuals who emigrated or died before their 6th birthday were excluded.

\section{Study variables-eating disorders}

Sweden: we defined and analyzed eating disorders grouped in two ways based on lifetime diagnoses of eating disorders in the Swedish NPR, the quality registers, and CDR before December 31, 2013: (1) AN, defined as any diagnosis of AN or atypical AN, identified with a Swedish ICD-9 code 307B and ICD-10 codes F50.0 or F50.1 in the NPR, or meeting DSM-IV-TR criteria for AN (307.1) or atypical AN (307.50, criteria 1 and 2) in the quality registers (other lifetime eating disorders could be present); (2) other eating disorder (OED), defined as having any eating disorder other than AN, identified with ICD-9 codes 307F and ICD-10 codes F50.2, F50.3, or F50.9 in the NPR, or meeting DSM-IV-TR criteria for any eating disorders other than AN (bulimia nervosa (307.51), atypical bulimia nervosa (307.50, criteria 3), or eating disorders not otherwise specified) in the quality registers. Both AN and OED were recorded if an individual received both diagnoses.

Denmark: probands were classified with AN (ICD-10: F50.0 or F50.1), or OED (ICD-10: F50.2, F50.3, F50.8, or F50.9) from the Danish NPR, PCRR, and CDR if they have at least one occurrence of an in- or outpatient eating disorder contact in PCRR or NPR or a registration of eating disorder as a cause of death in CDR, before January 1, 2017. Both AN and OED were recorded if an individual received both diagnoses.

\section{Study variables—schizophrenia}

Sweden: similar to previous studies [8, 29], we defined schizophrenia as $\geq 2$ inpatient or outpatient treatment contacts for schizophrenia or schizoaffective disorders from the Swedish NPR and the CDR, identified with ICD-8 codes 295.0-295.9 (except 295.5), Swedish ICD-9 codes 295A-295H (except 295F), 295W, 295X, V11A, and ICD-10 codes F200-F209 (except F207), F231, F232, F250-F252, F258, F259.

Denmark: we identified individuals with schizophrenia from the Danish PCRR, NPR, and CDR, with $\geq 2$ records of ICD-8 codes 295.0-295.9 (except 295.5), ICD-10 codes F20.0-F20.9 (except F20.7), F23.1, F23.2, F25.0-F25.2, F25.8, F25.9.

\section{Statistical analysis}

\section{Co-occurrence of eating disorders and schizophrenia within probands}

To evaluate the association between eating disorders (independent variable) and schizophrenia (dependent variable), logistic regressions were performed in the full proband cohort for each eating disorder subgroup, adjusted for sex and birth year (in quartiles), to obtain odds ratios (ORs) with $95 \%$ CIs in both countries separately. Sex stratified analyses were performed as well.

\section{Familial co-aggregation of eating disorders and schizophrenia}

We fit a logistic regression model for each of the six relative cohorts to evaluate the familial co-aggregation patterns of eating disorders (independent variable, from probands) and schizophrenia (dependent variable, from relatives) by estimating the ORs of schizophrenia in relatives of individuals (probands) with eating disorders compared with the same type of relatives of individuals (probands) without eating disorders. A higher OR in relatives with closer relatedness compared with more distant relatives suggests that eating disorders and schizophrenia share genetic and/or family environmental liabilities.

Because sex differences in the association between schizophrenia and eating disorders were not the main focus of this study, we combined males and females and adjusted for sex to increase the power in the analyses of familial coaggregation. In each model, we adjusted for birth year (in quartiles) and sex for both the proband and their relatives.

For all analyses, we used a cluster-robust (sandwich) estimator for standard error calculation, where clusters were identified via family identification numbers, which linked relatives to control for intrafamilial correlation within the data. Data management was performed using SAS, version 9.4 (SAS Institute, Inc.) in Sweden and Stata, version 15 in Denmark; analyses were performed using R, version 3.4.3 in Sweden and Stata, version 15 in Denmark. 


\section{Sensitivity analyses}

First, we repeated the analysis in the full sibling cohort with sex stratification. Next, we conducted tests to assess familial factors. In the full sibling cohorts, we repeated the analyses with additional adjustment for eating disorders in the full siblings (the person from whom we acquired information on schizophrenia status). ORs that remain in the same direction and significant further support the contribution of shared familial risk factors to eating disorders and schizophrenia (e.g., [30], explained in Supplementary method with Supplementary Figs. 1 and 2). Finally, since it is not uncommon for individuals to present with different eating disorder diagnoses across the course of their illness [31], we also performed the analysis with a stricter eating disorder category definition by evaluating individuals (probands) with exclusively AN or OED diagnoses (i.e., no indication of diagnostic crossover). We then repeated the analysis for all six relative cohorts.

\section{Results}

\section{Descriptive statistics}

Sweden: a total of 2,535,191 probands born 1977-2003 in Sweden were followed from their 6th birthday to
December 31, 2013. During the observation period, $12,863(0.51 \%)$ probands received at least one diagnosis of $\mathrm{AN}$, and $21,454(0.85 \%)$ probands received at least one diagnosis of OED. We identified $3146(0.12 \%)$ probands with schizophrenia.

Denmark: a total of 1,382,367 probands born 1984-2006 in Denmark were followed from their 6th birthday to December 31, 2016. During the observation period, 7120 $(0.52 \%)$ probands received at least one diagnosis of $\mathrm{AN}$, and $9731(0.70 \%)$ probands received at least one diagnosis of OED. We identified $6224(0.45 \%)$ probands with schizophrenia.

Females were more likely to receive eating disorder diagnoses, whereas the prevalence of schizophrenia was not significantly different between males and females in either country. The distribution of schizophrenia stratified by sex and eating disorder diagnosis among probands is shown in Table 1. Information about the relatives is detailed in Table 2.

\section{Co-occurrence of eating disorders and schizophrenia within probands}

Table 3 shows the ORs of schizophrenia for each eating disorder category and stratified by sex in the Swedish and Danish proband population. Individuals with AN had an increased risk of schizophrenia, reported as OR [95\% CI]:

Table 1 Sample sizes $(N)$ and prevalence $(\%)$ of the proband population in Sweden and Denmark.

\begin{tabular}{|c|c|c|c|c|c|c|c|c|}
\hline & \multicolumn{4}{|c|}{ Anorexia nervosa (AN) } & \multicolumn{4}{|c|}{ Other eating disorders (OED) } \\
\hline & \multicolumn{2}{|l|}{ Yes } & \multicolumn{2}{|l|}{ No } & \multicolumn{2}{|l|}{ Yes } & \multicolumn{2}{|l|}{ No } \\
\hline & Sweden & Denmark & Sweden & Denmark & Sweden & Denmark & Sweden & Denmark \\
\hline \multicolumn{9}{|l|}{ Eating disorders } \\
\hline No. of probands & 12,863 & 7120 & $2,522,328$ & $1,375,247$ & 21,454 & 9731 & $2,513,737$ & $1,372,636$ \\
\hline Female & 12,121 & 6599 & $1,225,367$ & 668,121 & 20,122 & 8672 & $1,217,366$ & 666,048 \\
\hline Male & 742 & 521 & $1,296,961$ & 707,126 & 1332 & 1059 & $1,296,371$ & 706,588 \\
\hline Prevalence, $\%$ & 0.51 & 0.52 & NA & NA & 0.85 & 0.70 & NA & NA \\
\hline Female & 0.98 & 0.98 & NA & NA & 1.63 & 1.29 & NA & NA \\
\hline Male & 0.06 & 0.07 & NA & NA & 0.10 & 0.15 & NA & NA \\
\hline \multicolumn{9}{|l|}{ Schizophrenia } \\
\hline No. of probands (\%) & $72(0.56)$ & $226(3.17)$ & $3074(0.12)$ & $5998(0.44)$ & $135(0.63)$ & $362(3.72)$ & $3011(0.12)$ & $5862(0.43)$ \\
\hline Female & $62(0.51)$ & $210(3.18)$ & $1129(0.09)$ & $2774(0.42)$ & $113(0.56)$ & $329(3.79)$ & $1078(0.09)$ & $2655(0.40)$ \\
\hline \multirow[t]{2}{*}{ Male } & $10(1.53)$ & $16(3.07)$ & $1945(0.15)$ & $3224(0.46)$ & $22(1.65)$ & $33(3.12)$ & $1933(0.15)$ & $3207(0.45)$ \\
\hline & \multicolumn{4}{|l|}{ Sweden } & \multicolumn{4}{|l|}{ Denmark } \\
\hline Prevalence $^{\mathrm{a}}, \%$ & \multicolumn{4}{|l|}{0.12} & \multicolumn{4}{|l|}{0.45} \\
\hline Female & \multicolumn{4}{|l|}{0.10} & \multicolumn{4}{|l|}{0.44} \\
\hline Male & \multicolumn{4}{|l|}{0.15} & \multicolumn{4}{|l|}{0.46} \\
\hline
\end{tabular}

NA not applicable.

${ }^{a}$ The prevalence of schizophrenia is the overall prevalence among proband population in Sweden and Denmark without stratification by eating disorder subgroup. 
Table 2 Sample sizes and prevalence of schizophrenia of the relatives in Sweden and Denmark.

\begin{tabular}{|c|c|c|c|c|c|c|}
\hline Characteristics & Parent & Full sibling & Grandparent & Half sibling & Aunt/uncle & Cousin \\
\hline \multicolumn{7}{|c|}{ Unique relatives $^{\mathrm{a}}, n$} \\
\hline Sweden & $2,329,145$ & $2,295,669$ & $2,665,509$ & 781,056 & $2,467,766$ & $3,166,256$ \\
\hline Denmark & $1,363,092$ & $1,218,780$ & $1,469,031$ & 410,966 & $1,221,060$ & $1,415,327$ \\
\hline \multicolumn{7}{|l|}{ Schizophrenia ${ }^{\mathrm{c}}, n$} \\
\hline Sweden $(\%)$ & $5482(0.24)$ & $3017(0.13)$ & $6877(0.26)$ & $2083(0.27)$ & $14,940(0.61)$ & $6394(0.20)$ \\
\hline Denmark $(\%)$ & $4446(0.33)$ & $5070(0.42)$ & $4125(0.28)$ & $3383(0.82)$ & $9774(0.80)$ & $6170(0.44)$ \\
\hline \multicolumn{7}{|c|}{ Analytical relative pairs ${ }^{\mathrm{b}}, n$} \\
\hline Sweden & $4,367,376$ & $3,369,781$ & $8,265,606$ & $1,432,934$ & $7,259,332$ & $13,905,022$ \\
\hline Denmark & $2,450,181$ & $1,703,887$ & $4,454,458$ & 703,569 & $3,427,089$ & $6,052,414$ \\
\hline
\end{tabular}

${ }^{a}$ Unique relatives, $n$ is the number of unique individuals in each relative cohort.

${ }^{\mathrm{b}}$ Analytical relative pairs, $n$ is the number of relative-index (SCZ outcome-ED exposure) pairs in analysis. Index person A with relative person B could also be analyzed as index person B with relative A if they fulfilled the requirement for each cohort.

${ }^{\mathrm{c}}$ Schizophrenia, $n$ is the number of unique individuals with schizophrenia in each relative cohort, and the percentage in parentheses is the prevalence of schizophrenia for each relative type.

Table 3 ORs for schizophrenia in probands with eating disorders.

\begin{tabular}{lll}
\hline & \multicolumn{1}{l}{ OR $(95 \%$ CI $)$ of Schizophrenia } \\
\cline { 2 - 3 } & Sweden & Denmark \\
\hline $\begin{array}{l}\text { AN } \\
\quad \begin{array}{l}\text { Total proband } \\
\text { population }\end{array}\end{array}$ & $5.66(4.46,7.19)$ & $6.17(5.38,7.08)$ \\
$\quad \begin{array}{l}\text { Female } \\
\quad \text { Male }\end{array}$ & $5.17(4.00,6.68)$ & $6.18(5.36,7.13)$ \\
$\begin{array}{l}\text { OED } \\
\text { Total proband } \\
\text { population } \\
\quad \text { Female } \\
\quad \text { Male }\end{array}$ & $10.99(5.88,20.53)$ & $6.08(3.68,10.06)$ \\
& $6.15(5.14,7.36)$ & $7.17(6.41,8.01)$ \\
& $5.47(4.50,6.64)$ & $7.22(6.42,8.12)$ \\
& $13.21(8.62,20.26)$ & $8.05(5.66,11.46)$ \\
\hline
\end{tabular}

The table shows ORs with $95 \%$ CI for schizophrenia in individuals with eating disorders compared with individuals without eating disorders.

OR odds ratio, $95 \%$ CI 95\% confidence interval, $A N$ anorexia nervosa, $O E D$ other eating disorders (non-AN eating disorders).

5.66 [4.46, 7.19] in Sweden and 6.17 [5.38, 7.08] in Denmark. The risk for schizophrenia in individuals with OED was also significantly increased (6.15 [5.14, 7.36] in Sweden and 7.17 [6.41, 8.01] in Denmark). We also examined the temporal diagnostic relationships between the first diagnoses for AN/OED and schizophrenia in our dataset: among individuals with both AN and schizophrenia, $80 \%$ in Sweden and $81 \%$ in Denmark of those received an AN diagnosis earlier than schizophrenia; among those with both OED and schizophrenia, 70\% in Sweden and $74 \%$ in Denmark received an OED diagnosis prior to schizophrenia.

There was no statistically significant difference between ORs for schizophrenia in individuals with OED and individuals with AN in either country (the OR for AN was included in the $95 \% \mathrm{CI}$ of OED and vice versa). Males with AN or OED had a significantly higher risk for schizophrenia than females in Sweden $\left(P\right.$ value $_{\mathrm{AN}}=0.035$, $P$ value $\left._{\mathrm{OED}}<0.001\right)$. However, this was not the case in the Danish sample.

\section{Familial co-aggregation of eating disorders and schizophrenia}

Figure 1 presents the ORs for schizophrenia in individuals (probands) with $\mathrm{AN}$ and their relatives compared with individuals (probands) without $\mathrm{AN}$ and their relatives. Full siblings of probands with AN were at increased risk of schizophrenia (OR [95\% CI]: 1.66 [1.21, 2.27] in Sweden and 1.47 [1.13, 1.92] in Denmark) compared with full siblings of probands without AN. Half siblings (including maternal and paternal half siblings) of probands with AN had an increased risk for schizophrenia in Denmark (1.42 [1.07, 1.87]), but the effect was not statistically significant in Sweden (1.33 [0.90, 1.95]). Grandparents of probands with AN had higher risk for schizophrenia (1.22 [1.02, 1.46]) in Sweden. However, this was not seen in Denmark $(0.91$ [0.69, 1.21]). No significant association was observed in other relatives (parents, uncles/aunts, and cousins). Overall, the magnitude of ORs attenuated with decreasing genetic relatedness among the youngest generation (full sibling $>$ half sibling $>$ cousin).

All relatives of probands with OED were at increased risk of schizophrenia; however, the effects among half siblings and uncles/aunts were not statistically significant in the Danish sample (Fig. 2). Overall, the magnitude of ORs attenuated with decreasing genetic relatedness. We also found a significant increase in the ORs for schizophrenia in 


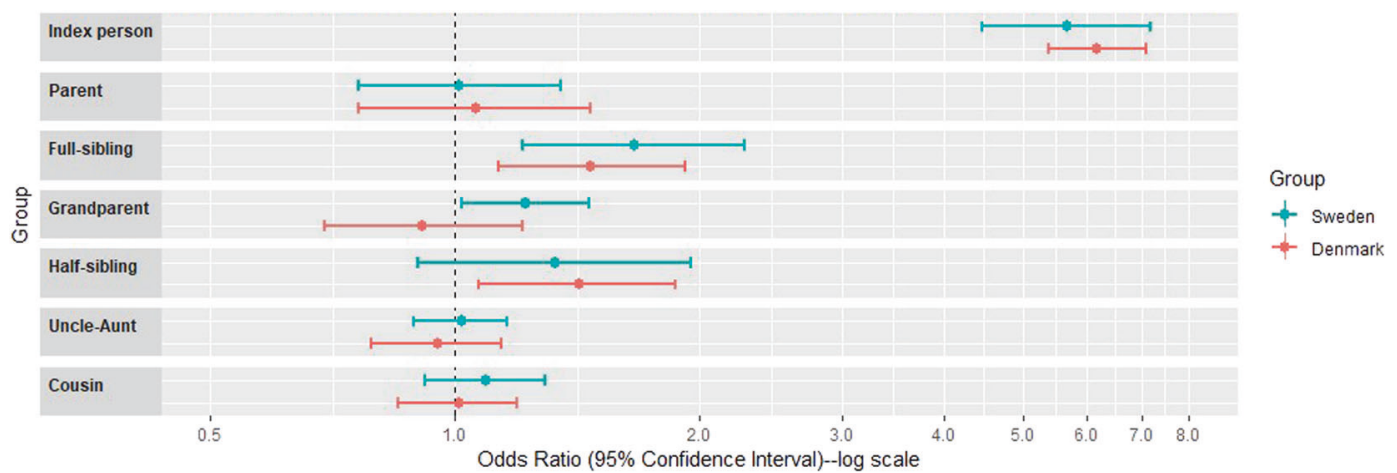

Fig. $1 \mathrm{OR}$ for SCZ in individuals with $\mathrm{AN}$ and their relatives, compared with individuals without $A N$ and their relatives. The forest plot shows ORs with $95 \%$ CI for SCZ in individuals with AN

and their relatives compared with individuals without AN and their relatives in Sweden and Denmark. OR odds ratio, 95\% CI 95\% confidence interval, SCZ schizophrenia, AN anorexia nervosa.

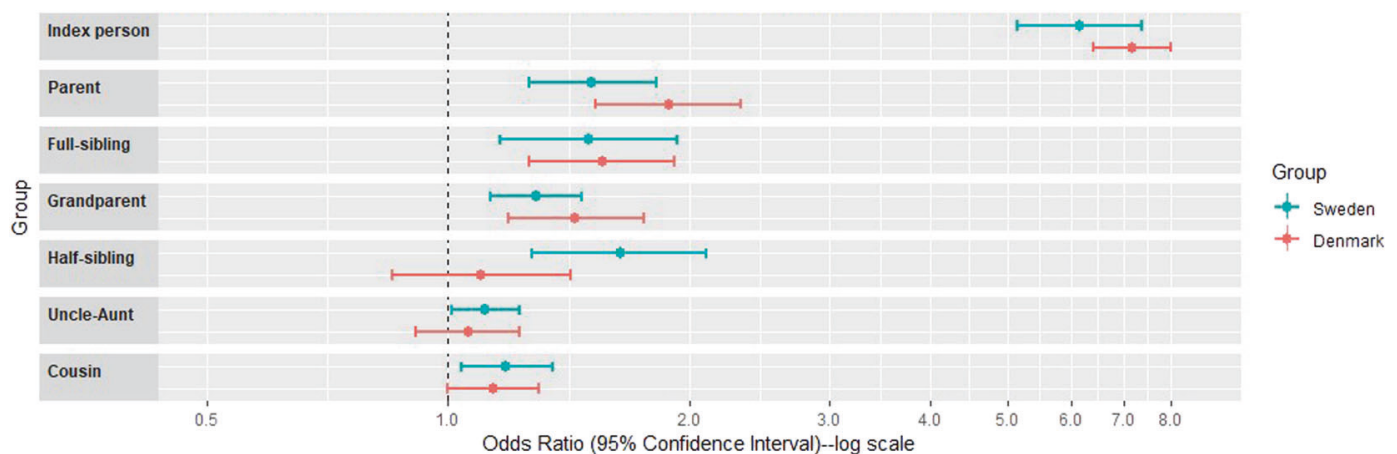

Fig. 2 OR for SCZ in individuals with OED and their relatives, compared with individuals without OED and their relatives. The forest plot shows ORs with 95\% CI for SCZ in individuals with OED and their relatives compared with individuals without OED and their

parents of probands with OED compared with those for parents of probands with AN (Supplementary Table 1).

\section{Sensitivity analyses}

The results for sex-specific analyses of familial coaggregation in full siblings are shown in Supplementary Table 2. In full siblings, males who had a sister with AN/OED had 1.5 times higher odds of schizophrenia compared with males who had a sister without AN/OED in both Sweden and Denmark. The results for other combinations were inconsistent between countries.

The results for analyses of full siblings with additional adjustment of AN/OED in the outcome person are shown in Supplementary Table 3. ORs for schizophrenia in full siblings of probands with eating disorders remained statistically significantly higher than 1 even after adjusting for the full siblings' eating disorders, supporting that familial factors partly contribute to the observed co-aggregation within relatives (explained in Supplementary method).

The results of analyses with a restricted eating disorder category definition are shown in Supplementary Table 4. These results are largely similar to the primary findings. relatives in Sweden and Denmark. OR odds ratio, 95\% CI 95\% confidence interval, SCZ schizophrenia, OED other eating disorders (non-AN eating disorders).

\section{Discussion}

We conducted a study of schizophrenia and eating disorder co-occurrence in a population of over 2.5 million probands in Sweden and 1.3 million probands in Denmark. This is the only known study exploring familial co-aggregation of schizophrenia and eating disorders. Our results demonstrate that individuals with any type of eating disorder had 6-7 times higher odds of schizophrenia than individuals without eating disorders. The pattern of familial co-aggregation of schizophrenia and eating disorders across different relative types stratified by AN and OED indicated different mechanisms for shared factors in schizophrenia and AN vs. schizophrenia and OED.

A positive genetic correlation between schizophrenia and AN has been found in previous studies [10,11], which is in line with our findings of elevated risk of schizophrenia in probands with $\mathrm{AN}$, indicating that genetic factors contribute to the co-occurrence of AN and schizophrenia. However, the observed association of AN and schizophrenia in probands and full siblings (and likely half siblings) but not parents is difficult to interpret. One potential explanation could be the low fecundity observed in schizophrenia [32]. 
Since we expanded our cohorts from probands to their parents, individuals with schizophrenia without children would not be represented in the study population, which might lead to the lack of observed association between probands with $\mathrm{AN}$ and parents with schizophrenia. However, the association between this type of pair (probands-parents) in schizophrenia and OED has been detected, which indicates that the low fecundity for schizophrenia was not the only factor. Shared childhood environmental effects could also account for some of the association. Although shared environmental factors do not contribute to liability to AN based on a previous twin study [33], they do comprise $11 \%$ of the liability to schizophrenia [9]. Another potential explanation could be de novo mutations, which increase with paternal age and have been associated with increased risk for both schizophrenia and AN $[34,35]$. In that case, the association would diminish in full siblings after adjusting for paternal age. However, we did not observe a significant change in the adjusted model (Supplementary Table 5).

For added context, we also tested in our full sibling cohort for the risk of other psychiatric disorders (obsessivecompulsive disorder, major depression, and anxiety disorders), which have demonstrated positive genetic correlations with AN in previous studies $[11,36]$. Interestingly, the magnitude of ORs for the other psychiatric disorders and AN are largely consistent with the genetic correlation found between them (Supplementary Table 6). Moreover, a recent study revealed the pleiotropy effects of contributing loci associated with eight different psychiatric disorders, identifying three groups of inter-related disorders [37]. Although AN and schizophrenia still belong to different clusters, a number of pleiotropic loci were found to be associated with both disorders [37]. Together with results from Supplementary Table 6 , these findings indicate a broader domains underlying genetic risk to psychopathology, which are in conflict with the traditional categorical taxonomies of mental disorders [38].

In terms of the association between schizophrenia and OED, significant evidence for linkage to marker D10S1423 on 10p has been observed in both schizophrenia and bulimia nervosa [39, 40]. Furthermore, increased schizophrenia polygenic risk scores have been found to be associated with greater odds of having binge-eating behaviors [41]. Similar to previous findings of association between having a firstdegree relative with schizophrenia and the lifetime risk for bulimia nervosa from survey data [18], our results show consistent association of the risk for schizophrenia in firstdegree relatives of probands with OED. By comparing ORs across different types of relatives, our study provided deeper exploration of the origins of the familial co-aggregation. If the familial co-aggregation was primarily due to genetic factors, ORs would be expected to decrease based on the degree of relatedness. We did observe a tendency for the magnitude of ORs for schizophrenia associated with OED to attenuate with decreasing genetic relatedness. This suggests a common genetic contribution for schizophrenia and OED, and supports the need for studies to further identify the shared genetic factors.

Limitations of this study include under-detection of individuals with schizophrenia or eating disorders diagnosis. Individuals might have an outpatient diagnosis for which the records were only available after 2001 in Sweden. Records from private caregivers also cannot be completely captured in both countries. Since it is only possible to follow individuals until the latest update of the data, we cannot capture recent diagnoses or presentations later in life. Under-detection would likely lead to underestimation of the effects. Nevertheless, if relatives of people with a psychiatric diagnosis are more likely to have healthcare exposures, they may have a greater chance of receiving diagnoses compared with relatives of people without a psychiatric diagnosis, which might lead to an overestimation of the effect. Misdiagnosis is potentially a concern if, for example, feeding behavior changes as a result of psychotic symptoms and is incorrectly attributed to an eating disorder. However, $<1 \%$ of all cases in the Swedish quality registers for eating disorders were later determined to be erroneous, suggesting that this is unlikely to impact our results [23]. On a related note, it is a limitation of current clinical diagnostic systems that they only permit separate diagnoses of schizophrenia and eating disorders despite the fact that these may co-occur in the same individual due to a singular disease process. Additional research to resolve the origin of this putative comorbidity is needed. Furthermore, the zygosity of twins was not available in Danish databases to which we had access. Consequently, we included dizygotic twins as full siblings in Sweden, but not in Denmark. However, this did not appear to have a strong influence on the final results. In addition, since people with AN might later develop OED or vice versa, we performed sensitivity analyses using strict definitions of AN and OED. No appreciable differences emerged (Supplementary Table 4). Another potential bias could be differing diagnostic practices by clinicians for both eating disorders and schizophrenia in Sweden and Denmark, which could increase heterogeneity. Previous validation studies have however confirmed a low number of false-positive diagnoses for schizophrenia [42-44]. We also observed that the overall prevalence of schizophrenia was higher in Denmark than in Sweden. Since the oldest individuals in the Swedish proband cohort were 36 vs. 32 in Denmark, a higher proportion of probands should have passed through the peak ages at of onset, driving the prevalence in the opposite of the observed direction. We were unable to identify the cause of this discrepancy, but note that it has been reported previously [45]. 
The main strengths of our study are studying large-scale population-based cohorts, the national coverage of all available psychiatric contacts, large sample size for both disorders, and relatively consistent findings in two countries, strengthening both reliability and generalizability. Restricting to at least two records of schizophrenia for case definition also reduced potential bias due to misclassification and increased certainty of the diagnoses.

By studying two large population-based cohorts, we found that individuals with AN or OED were more likely to have schizophrenia than people without these eating disorders. Shared risk factors exist between schizophrenia and eating disorders, particularly OED. Additional studies such as genome-wide association studies of bulimia nervosa and binge-eating disorder and calculation of genetic correlations between schizophrenia and bulimia/binge-eating disorder are needed to distinguish the shared and specific genetic and environmental risk factors for schizophrenia and eating disorders. Clinicians should be mindful of comorbidity between eating disorders and schizophrenia as it may inform case conceptualization and treatment decisions. For example, AN treatment with the antipsychotic olanzapine [46] might have differential outcomes in individuals with family histories of schizophrenia or high schizophrenia genetic risk. Future studies will address how family history and genetic risk for schizophrenia influence the clinical course of eating disorders.

Acknowledgements RZ was supported by Chinese Scholarship Council (CSC201700260258) and by the Swedish Medical Association \#777621 (PI: SB). LVP was supported by the Lundbeck Foundation (grant no R276-2017-4581). CMB acknowledges funding from the Swedish Research Council (VR Dnr: 538-2013-8864).

\section{Compliance with ethical standards}

Conflict of interest CMB has served on scientific advisory boards for Shire, serves as a consultant for Idorsia, and received royalties from Pearson (all outside the submitted work). HL has served as a speaker for Evolan Pharma and Shire and has received research grants from Shire (all outside the submitted work).

Publisher's note Springer Nature remains neutral with regard to jurisdictional claims in published maps and institutional affiliations.

\section{References}

1. World Health Organization. Mental disorders. 2017. http://www. who.int/mediacentre/factsheets/fs396/en/.

2. Beidel DC, Bulik CM, Stanley MA. Abnormal psychology. 3rd ed. NJ: Pearson Education; 2014. p. 621.

3. Arcelus J, Mitchell AJ, Wales J, Nielsen S. Mortality rates in patients with anorexia nervosa and other eating disorders. A meta-analysis of 36 studies. Arch Gen Psychiatry. 2011;68:724-31.

4. Swinbourne JM, Touyz SW. The co-morbidity of eating disorders and anxiety disorders: a review. Eur Eat Disord Rev. 2007;15:253-74.
5. Bulik CM, Reichborn-Kjennerud T. Medical morbidity in binge eating disorder. Int J Eat Disord. 2003;34:S39-46.

6. Hilker R, Helenius D, Fagerlund B, Skytthe A, Christensen K, Werge TM, et al. Heritability of schizophrenia and schizophrenia spectrum based on the Nationwide Danish Twin Register. Biol Psychiatry 2018;83:492-8.

7. Klump KL, Suisman JL, Burt SA, McGue M, Iacono WG. Genetic and environmental influences on disordered eating: an adoption study. J Abnorm Psychol. 2009;118:797-805.

8. Lichtenstein P, Yip BH, Bjork C, Pawitan Y, Cannon TD, Sullivan $\mathrm{PF}$, et al. Common genetic determinants of schizophrenia and bipolar disorder in Swedish families: a population-based study. Lancet. 2009;373:234-9.

9. Sullivan PF, Kendler KS, Neale MC. Schizophrenia as a complex trait: evidence from a meta-analysis of twin studies. Arch Gen Psychiatry. 2003;60:1187-92.

10. Bulik-Sullivan B, Finucane HK, Anttila V, Gusev A, Day FR, Loh $\mathrm{PR}$, et al. An atlas of genetic correlations across human diseases and traits. Nat Genet. 2015;47:1236-41.

11. Duncan L, Yilmaz Z, Gaspar H, Walters R, Goldstein J, Anttila V, et al. significant locus and metabolic genetic correlations revealed in genome-wide association study of anorexia nervosa. Am J Psychiatry. 2017;174:850-8.

12. Bou Khalil R, Hachem D, Richa S. Eating disorders and schizophrenia in male patients: a review. Eat Weight Disord. 2011;16: e150-6.

13. Striegel-Moore RH, Garvin V, Dohm FA, Rosenheck RA. Psychiatric comorbidity of eating disorders in men: a national study of hospitalized veterans. Int J Eat Disord. 1999;25:399-404.

14. Lyketsos GC, Paterakis P, Beis A, Lyketsos CG. Eating disorders in schizophrenia. Br J Psychiatry 1985;146:255-61.

15. Rasmussen AR, Reich D, Lavoie S, Li E, Hartmann JA, McHugh $\mathrm{M}$, et al. The relation of basic self-disturbance to self-harm, eating disorder symptomatology and other clinical features: exploration in an early psychosis sample. Early Interv Psychiatry. 2019:1-8. https://doi.org/10.1111/eip.12850.

16. Cannon M, Walsh E, Hollis C, Kargin M, Taylor E, Murray RM, et al. Predictors of later schizophrenia and affective psychosis among attendees at a child psychiatry department. Br J Psychiatry. 2001;178:420-6.

17. Solmi F, Melamed D, Lewis G, Kirkbride JB. Longitudinal associations between psychotic experiences and disordered eating behaviours in adolescence: a UK population-based study. Lancet Child Adolesc Health. 2018;2:591-9.

18. DeVylder JE, Lukens EP. Family history of schizophrenia as a risk factor for axis I psychiatric conditions. J Psychiatr Res. 2013; 47:181-7.

19. Ludvigsson JF, Almqvist C, Bonamy AK, Ljung R, Michaelsson K, Neovius M, et al. Registers of the Swedish total population and their use in medical research. Eur J Epidemiol. 2016;31:125-36.

20. Axelsson O. The Swedish medical birth register. Acta Obstet Gynecol Scand. 2003;82:491-2.

21. Ludvigsson JF, Andersson E, Ekbom A, Feychting M, Kim JL, Reuterwall C, et al. External review and validation of the Swedish national inpatient register. BMC Public Health. 2011;11:450.

22. American Psychiatric Association. Diagnostic and statistical manual of mental disorders: DSM-IV-TR. Washington, DC: American Psychiatric Association; 2000.

23. Birgegard A, Bjorck C, Clinton D. Quality assurance of specialised treatment of eating disorders using large-scale Internetbased collection systems: methods, results and lessons learned from designing the Stepwise database. Eur Eat Disord Rev. 2010;18:251-9.

24. Pedersen CB, Gotzsche H, Moller JO, Mortensen PB. The Danish Civil Registration System. A cohort of eight million persons. Dan Med Bull. 2006;53:441-9. 
25. Knudsen LB, Olsen J. The Danish Medical Birth Registry. Dan Med Bull. 1998;45:320-3.

26. Statistics Denmark. Documentation variables (Times). 2019. https://www.dst.dk/da/Statistik/dokumentation/Times/.

27. Mors O, Perto GP, Mortensen PB. The Danish Psychiatric Central Research Register. Scand J Public Health. 2011;39:54-7.

28. Lynge E, Sandegaard JL, Rebolj M. The Danish National Patient Register. Scand J Public Health. 2011;39:30-3.

29. Schizophrenia Working Group of the Psychiatric Genomics Consortium, Ripke S, Neale BM, Corvin A, JTR Walters, Farh K$\mathrm{H}$, et al. Biological insights from 108 schizophrenia-associated genetic loci. Nature. 2014;511:421.

30. Yao S, Kuja-Halkola R, Thornton LM, Runfola CD, D'Onofrio BM, Almqvist C, et al. Familial liability for eating disorders and suicide attempts: evidence from a Population Registry in Sweden. JAMA Psychiatry. 2016;73:284-91.

31. Schaumberg K, Jangmo A, Thornton LM, Birgegård A, Almqvist $\mathrm{C}$, Norring $\mathrm{C}$, et al. Patterns of diagnostic transition in eating disorders: a longitudinal population study in Sweden. Psychol Med. 2018;49:819-27.

32. Power RA, Kyaga S, Uher R, MacCabe JH, Långström N, Landen $\mathrm{M}$, et al. Fecundity of patients with schizophrenia, autism, bipolar disorder, depression, anorexia nervosa, or substance abuse vs their unaffected siblings. JAMA Psychiatry. 2013;70:22-30.

33. Bulik CM, Thornton LM, Root TL, Pisetsky EM, Lichtenstein P, Pedersen NL. Understanding the relation between anorexia nervosa and bulimia nervosa in a Swedish national twin sample. Biol Psychiatry. 2010;67:71-7.

34. Javaras KN, Rickert ME, Thornton LM, Peat CM, Baker JH, Birgegård $\mathrm{A}$, et al. Paternal age at childbirth and eating disorders in offspring. Psychol Med. 2017;47:576-84.

35. Sipos A, Rasmussen F, Harrison G, Tynelius P, Lewis G, Leon DA, et al. Paternal age and schizophrenia: a population based cohort study. BMJ. 2004;329:1070.

36. Watson HJ, Yilmaz Z, Thornton LM, Hübel C, Coleman JRI, Gaspar HA, et al. Genome-wide association study identifies eight risk loci and implicates metabo-psychiatric origins for anorexia nervosa. Nat Genet. 2019;51:1207-14.

37. Lee PH, Anttila V, Won H, Feng Y-CA, Rosenthal J, Zhu Z, et al. Genomic relationships, novel loci, and pleiotropic mechanisms across eight psychiatric disorders. Cell. 2019;179:1469-82.e11.

38. Kotov R, Krueger RF, Watson D, Achenbach TM, Althoff RR, Bagby RM, et al. The hierarchical taxonomy of psychopathology (HiTOP): a dimensional alternative to traditional nosologies. J Abnorm Psychol. 2017;126:454-77.

39. Bulik C, Devlin B, Bacanu S-A, Thornton L, Klump K, Fichter M, et al. Significant linkage on chromosome 10p in Families with bulimia nervosa. Am J Hum Genet. 2003;72:200-7.

40. Faraone SV, Meyer J, Matise T, Svrakic D, Pepple J, Malaspina $\mathrm{D}$, et al. Suggestive linkage of chromosome 10p to schizophrenia is not due to transmission ratio distortion. Am J Med Genet. 1999;88:607-8.

41. Solmi F, Mascarell MC, Zammit S, Kirkbride JB, Lewis G. Polygenic risk for schizophrenia, disordered eating behaviours and body mass index in adolescents. Br J Psychiatry. 2019;215:428-33.

42. Dalman C, Broms J, Cullberg J, Allebeck P. Young cases of schizophrenia identified in a national inpatient register. Soc Psychiatry Psychiatr Epidemiol. 2002;37:527-31.

43. Ekholm B, Ekholm A, Adolfsson R, Vares M, Osby U, Sedvall $\mathrm{GC}$, et al. Evaluation of diagnostic procedures in Swedish patients with schizophrenia and related psychoses. Nord J Psychiatry. 2005;59:457-64.

44. Jakobsen KD, Frederiksen JN, Hansen T, Jansson LB, Parnas J, Werge T. Reliability of clinical ICD-10 schizophrenia diagnoses. Nord J Psychiatry. 2005;59:209-12.

45. Liang H, Olsen J, Yuan W, Cnattingus S, Vestergaard M, Obel C, et al. Early life bereavement and schizophrenia: a nationwide cohort study in Denmark and Sweden. Medicine. 2016;95:e2434.

46. Attia E, Steinglass JE, Walsh BT, Wang Y, Wu P, Schreyer C, et al. Olanzapine versus placebo in adult outpatients with anorexia nervosa: a randomized clinical trial. Am J Psychiatry. 2019;176:449-56. 